\title{
Algoritmo Simulated Annealing Modificado (ASAM) para Minimizar Peso en Cerchas Planas con Variables Discretas
}

\section{Modified Simulated Annealing Algorithm (MSAA) for Plane Trusses Weight Minimization with Discrete Variables}

\author{
DOI: http://dx.doi.org/10.17981/ingecuc.12.2.2016.01
}

Artículo de Investigación Científica - Fecha de Recepción: 22 de Octubre de 2015 - Fecha de Aceptación: 7 de Marzo de 2016

\author{
Carlos Millán Páramo* \\ Departamento de Ingeniería Civil, Universidad de Sucre \\ Sincelejo, Colombia. carlos.millan@unisucre.edu.co \\ Euriel Millán Romero \\ Departamento de Ingeniería Agrícola, Universidad de Sucre \\ Sincelejo, Colombia. euriel.millan@unisucre.edu.co
}

Cómo citar este artículo:

C. Millán y E. Millán, "Algoritmo Simulated Annealing Modificado ASAM para Minimizar Peso en Cerchas Planas con Variables

Discretas”, INGE CUC, vol. 12, No.2, pp. 9-16, 2016. DOI: http://dx.doi.org/10.17981/ingecuc.12.2.2016.01

\begin{abstract}
Resumen- El objetivo de este trabajo es emplear un algoritmo de optimización estocástico ASAM (Algoritmo Simulated Annealing Modificado) para optimizar (minimización de peso) cerchas planas con variables discretas. ASAM se basa en el proceso de enfriamiento de metales empleado en el Simulated Annealing (SA) clásico, pero posee tres características fundamentales (exploración preliminar, paso de búsqueda y probabilidad de aceptación) que lo diferencian de este. Para evaluar y validar el desempeño de ASAM se abordaron tres problemas de minimización de peso en cerchas planas con variables discretas reportados en la literatura especializada, y los resultados son comparados con los obtenidos por otros autores empleando diferentes algoritmos de optimización. Se concluyó que el algoritmo ASAM presentado en este estudio puede ser utilizado eficazmente en la minimización de peso de cerchas planas.
\end{abstract}

Palabras clave- Algoritmo simulated annealing modificado, optimización, variables discretas, cercha plana, minimización de peso.

\begin{abstract}
The aim of this study is to use stochastic optimization algorithm MSAA (Modified Simulated Annealing Algorithm) for trusses plane optimization (weight minimization) with discrete variables. MSAA is based on the cooling process of metal used in the Simulated Annealing (SA) classic, but it has three fundamental characteristics (preliminary exploration, search step and acceptance probability) that differentiate this. To evaluate and validate the MSAA performance were studied three problems plane trusses weight minimization with discrete variables reported in the literature and the results are compared with those obtained by other authors using different optimization algorithms. It is concluded that the MSAA algorithm presented in this study can be effectively used in the weight minimization of truss structures.
\end{abstract}

Keywords- Modified simulated annealing algorithm, optimization, discrete variables, plane truss, weight minimization.

*Autor de Correspondencia: carlos.millan@unisucre.edu.co 


\section{INTRODUCCIÓN}

Los algoritmos de optimización son desarrollados con objeto de maximizar o minimizar funciones, y se obtiene como solución un conjunto de variables discretas o continuas que satisfacen simultáneamente las limitaciones que impone el problema. En muchos problemas de ingeniería, las variables de diseño son discretas debido a la disponibilidad de los elementos en tamaños estándar.

Diversos estudios de optimización con variables discretas han sido reportados empleando algoritmos de optimización populares como Algoritmos Genéticos (AG), Recocido Simulado (Simulated Annealing: SA), Optimización con Colonia de Hormigas (Ant Colony Optimization: ACO), Optimización con Enjambre de Partículas (Particle Swarm Optimization: PSO), Búsqueda Armónica (Harmony Search: HS), entre otros.

El algoritmo HS desarrollado por Geem [1] se basa en el proceso de interpretación musical que se produce cuando un músico busca un mejor estado de la armonía. SA fue propuesto por Kirpatrick [2] y trata de simular el proceso de enfriamiento de metales para resolver problemas de optimización. El AG es un algoritmo que imita el proceso de evolución del hombre y fue propuesto por Holland [3] y Goldberg [4]. El algoritmo ACO está inspirado en el comportamiento de alimentación exhibida por las colonias de hormigas reales y fue propuesto por Dorigo [5] para la solución de problemas de optimización combinatoria. La optimización por enjambre de partículas que se basa en el comportamiento de los animales en busca de alimentos fue desarrollado por primera vez por Kennedy y Eberhart [6].

La optimización estructural (minimización de peso) es un problema con múltiples mínimos locales, susceptibles de solución a través de métodos estocásticos diseñado para identificar mínimos globales. Por lo tanto, en este trabajo se propone emplear el Algoritmo Simulated Annealing Modificado (ASAM) [7], debido a su notable desempeño en comparación con técnicas como Harmony Search, Algoritmos Genéticos y PSO-DE (Particle swarm optimization-differential evolution), entre otras. Para mayores detalles se recomienda consultar [7].

En su primera parte este trabajo presenta la descripción del problema de optimización estructural. Seguidamente se describe brevemente el algoritmo ASAM, sus fundamentos y los parámetros que lo controlan. Finalmente, para demostrar la eficacia del algoritmo se muestran los problemas numéricos y los resultados obtenidos y se comparan con resultados de la literatura internacional.

\section{Descripción del Problema}

Muchos problemas en la ingeniería tienen múltiples soluciones, y seleccionar el más adecuado puede ser una tarea importante. En problemas de optimización de cerchas planas con variables discretas, la tarea principal es seleccionar una sección transversal óptima de los elementos de una lista admisible de secciones estándar que minimizan el peso de la estructura al tiempo que satisface las restricciones de diseño [8]. La forma de restricción del problema de optimización se puede expresar como

$$
\begin{gathered}
\text { Minimizar: } W(A)=\sum_{i=1}^{n} \gamma_{i} A_{i} L_{i}(x) \\
\text { Sujeto a: } \delta_{\min } \leq \delta_{i} \leq \delta_{\max }, i=1,2, \ldots, m \\
\sigma_{\min } \leq \sigma_{i} \leq \sigma_{\max }, i=1,2, \ldots, n
\end{gathered}
$$

Donde $A_{i}, L_{i}$, y $\gamma_{i}$ son la sección transversal, longitud y densidad por unidad de volumen del elemento $i$, respectivamente; $W(A)$ es el peso de la estructural que se minimiza; $n$ es el número total de elementos; $\sigma_{i} \mathrm{y} \delta_{i}$ son los esfuerzos en los miembros y deflexiones en los nodos permitidos, respectivamente.

En problemas de optimización discretos, el vector A representa la sección del elemento seleccionado de la lista de perfiles permisible. La lista $\mathbf{L}$ incluye todos los valores discretos disponibles dispuestas en secuencias ascendentes y puede expresarse como sigue:

$$
L_{i}=\left\{A_{1}, A_{2}, \ldots, A_{i}, \ldots, A_{p}\right\}, 1 \leq \mathbf{j} \leq p
$$

Donde $p$ es el número de secciones disponibles.

\section{III.Algoritmo Simulated Annealing Modificado}

Antes de sintetizar las características de ASAM vale la pena describir brevemente el funcionamiento del Simulated Annealing básico. SA comienza con un cierto estado $S$. A través de un proceso único crea un estado vecino $S^{\prime}$ al estado inicial. Si la energía o la evaluación del estado $S^{\prime}$ son menores que el estado $S$, cambia el estado $S$ por $S^{\prime}$. Si la evaluación de $S^{\prime}$ es mayor que la de $S$ puede estar empeorando, por lo que elige $S^{\prime}$ en vez de $S$ con una cierta probabilidad que depende de las diferencias en las evaluaciones y la temperatura del sistema T. La probabilidad de aceptar un peor estado se calcula mediante la siguiente ecuación:

$P(\Delta f, T)=\mathrm{e}^{(\Delta f / T)}$

Donde:

$P$ : $\quad$ Probabilidad de aceptar el nuevo estado.

$\Delta f: \quad$ Diferencia de las evaluaciones de la función para cada estado.

$T: \quad$ Temperatura del sistema.

e: Número de Euler.

Inicialmente, con valores grandes para T frecuentemente se aceptan soluciones con un mayor valor de función objetivo; a medida que el valor de T disminuye, tal tipo de soluciones raramente se aceptan, y cuando $\mathrm{T}$ se acerca a cero solo se aceptan aquellas soluciones que mejoran la anterior. Varios estudios teóricos demuestran que si T decrece con la suficien- 
te lentitud, el proceso converge a la solución óptima [8]. La función para reducción de temperatura más utilizada es: $\mathrm{T}_{\mathrm{k}+1}=\mathrm{T}_{\mathrm{k}} \cdot \mathrm{\alpha}$, donde $\mathrm{T}_{\mathrm{k}+1}$ es el nuevo valor ajustado de $\mathrm{T}, \mathrm{T}_{\mathrm{k}}$ corresponde al previo valor de $\mathrm{T}$ y a es una constante que está comprendida en el intervalo [0.8-0.99].

SA comienza con una solución inicial escogida aleatoriamente en el espacio de búsqueda y la compara con otra que también se selecciona estocásticamente en el espacio de búsqueda, lo cual afecta al algoritmo cuando se tienen funciones altamente dimensionales y modales generando mayores tiempos de búsqueda y soluciones subóptimas. Además, la probabilidad de aceptación de una solución peor se encuentra en un intervalo de entre 0 y 1 , lo cual causa que a temperaturas iniciales el algoritmo acepte un gran número de soluciones de peor calidad (aumentando el riesgo de quedar atrapado en un óptimo local).

En este contexto el algoritmo ASAM tiene 3 características fundamentales que lo hacen diferente respecto al Simulated Annealing básico. Dichas características son las siguientes:

\section{A. Exploración preliminar}

En esta etapa el algoritmo realiza una exploración en todo el espacio de búsqueda que viene dado por la siguiente matriz:

$$
\begin{array}{ll}
X_{P x N}=I_{P x N} X_{\min }+\operatorname{rand}_{P x N}\left(X_{\max }-X_{\min }\right) \\
\begin{array}{ll}
\text { Donde } \\
P:
\end{array} & \begin{array}{l}
\text { Número de puntos (estados) que se } \\
\text { desean en el espacio de búsqueda. }
\end{array} \\
N: & \text { Número de dimensiones del problema. } \\
I_{P x N}: & \text { Matriz identidad de tamaño PxN. } \\
& \text { Límite inferior del problema. } \\
X_{\min }: & \text { Límite superior del problema. } \\
\operatorname{rand}_{P x N}: & \begin{array}{l}
\text { Matriz P X N de números aleatorios } \\
\text { (aleatoriedad pura) entre 0 y 1. }
\end{array}
\end{array}
$$

Para comenzar el proceso de optimización con ASAM se evalúan todos los puntos generados con la ecuación 6 mediante la función objetivo del problema y se escoge el que tenga menor valor (en el caso de estar buscando el valor mínimo de la función) como punto inicial de la búsqueda.

\section{B. Paso de búsqueda}

A partir del punto inicial determinado en la etapa anterior se genera un paso de búsqueda para determinar el estado vecino. Este paso depende de un radio de acción que se reduce gradualmente a medida que desciende la temperatura del sistema. Es decir, cuando el algoritmo está en determinada temperatura, con radio de acción definido por la ecuación 7 , la transición del punto inicial al nuevo punto (paso de búsqueda) se realiza mediante la adición de nú- meros aleatorios que están comprendidos entre cero y el valor del radio. Esto permite que el algoritmo realice una exploración global a temperaturas altas y una exploración local a temperaturas bajas, dando un equilibrio entre la exploración y la explotación del algoritmo.

$$
\begin{aligned}
& R_{i+1}=R_{i} \cdot a \\
& \text { Donde } \\
& R_{i}: \quad \text { Radio inicial ciclo. } \\
& a ; \quad \text { Coeficiente de reducción del radio. }
\end{aligned}
$$

\section{Probabilidad de aceptación}

En esta propuesta la probabilidad de aceptación de una solución (estado) peor viene dada por

$$
\mathrm{P}=\frac{1}{1+\mathrm{e}^{(\Delta / \mathrm{T})}}
$$

$P$ : $\quad$ Probabilidad de aceptar el nuevo estado.

$\Delta f: \quad$ Diferencia de las evaluaciones de la función para cada estado.

$T: \quad$ Temperatura del sistema.

e: Número de Euler.

Esta probabilidad se encuentra en un intervalo entre 0 y $1 / 2$, lo cual permite al algoritmo tener un rango menor de aceptación de peores soluciones.

En resumen, las 3 modificaciones propuestas en ASAM tienen la finalidad de mejorar la exploración inicial, permitir un balance entre exploración inicial y final y controlar la convergencia en la etapa final de búsqueda.

\section{III.Problemas Numéricos}

Los siguientes 3 problemas clásicos de diseño con variables discretas fueron utilizados para investigar la exactitud numérica, eficiencia y validación del algoritmo ASAM:

- Cercha plana de 10 barras

- Cercha plana de 15 barras

- Cercha plana de 52 barras

Se realizaron 100 corridas independientes del algoritmo para cada problema. Los mejores y peores diseños y el peso de la estructura obtenidos con ASAM son reportados en las Tablas 1, 2 y 4 . La implementación del algoritmo fue realizada en Matlab $\AA$, bajo el sistema operativo Windows 7, y el equipo utilizado fue un Intel Core i7-2.4 GHz, 8GB (RAM).

\section{A. Cercha plana de 10 barras}

La Fig. 1 muestra la geometría y las condiciones de carga de la cercha que consta de 10 elementos. Esta estructura es un ejemplo estándar y ha sido utilizada por muchos investigadores, incluyendo Capriles [10], Camp [11,12], Barbosa [13], Li [14], Sonmez, [8] y Dede [15]. 


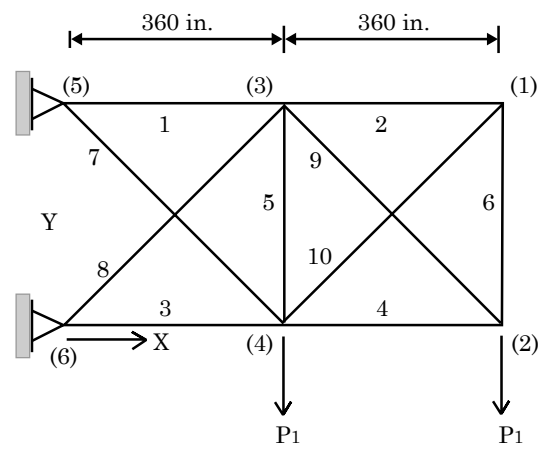

Fig. 1. Cercha plana de 10 barras Fuente: Camp [12]

Los miembros están fabricados de un material con módulo de elasticidad E=10000 ksi y densidad por unidad de volumen lb/in ${ }^{3}$. Los desplazamientos de los nodos libres no deben exceder \pm 2 in en dirección vertical y horizontal; además, los esfuerzos admisibles tanto a tensión y compresión no deben superar 20 ksi y P1=100 kips. Las secciones transversales de todos los 10 miembros son seleccionadas de la siguiente lista $\mathrm{L}=\{1,62 ; 1,80 ; 1,99 ; 2,13 ; 2,38 ; 2,62$; 2,$63 ; 2,88 ; 2,93 ; 3,09 ; 3,13 ; 3,38 ; 3,47 ; 3,55 ; 3,63$; 3,$84 ; 3,87,3,88 ; 4,18 ; 4,22 ; 4,49 ; 4,59 ; 4,80 ; 4,97$; 5,$12 ; 5,74 ; 7,22 ; 7,97 ; 11,5 ; 13,5 ; 13,9 ; 14,2 ; 15,5 ; 16,0$; $16,9 ; 18,8 ; 19,9 ; 22,0 ; 22,9 ; 26,5 ; 30,0 ; 33,5\}\left(\right.$ in $\left.^{2}\right)$.

La mejor solución encontrada por ASAM es [33,5; $1,62 ; 22,9 ; 14,2 ; 1,62 ; 1,62 ; 7,97 ; 22,9 ; 22,0 ; 1,62] \mathrm{in}^{2}$ y un peso mínimo de $5490.74 \mathrm{lb}$. La gráfica de convergencia se muestra en la Fig. 2, donde se observa cómo el algoritmo va encontrando el peso óptimo a medida que desciende la temperatura del sistema.

En el anexo Tabla 1 se muestran la mejor y peor solución encontrada por ASAM y son comprados con los resultados encontrados por los autores mencionados anteriormente utilizando diferentes algoritmos de optimización.

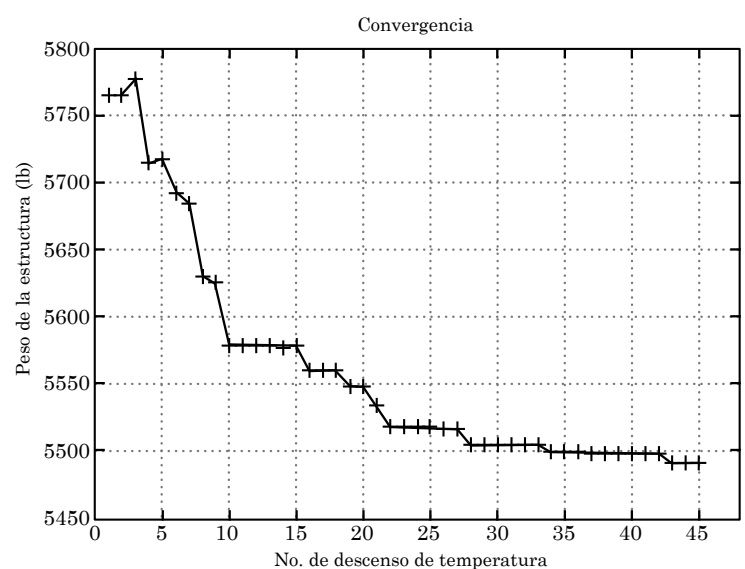

Fig. 2. Convergencia cercha plana de 10 barras Fuente: Autores

\section{B. Cercha plana de 15 barras}

Zhang [16], Li [14], Sabohur [17] y Dede [15] han optimizado este problema mostrado en la Fig. 3. La densidad del material es $7.8 \times 10-6 \mathrm{~kg} / \mathrm{mm}^{3}$ y el módulo de elasticidad es $200000 \mathrm{MPa}$. Los esfuerzos permitidos son $\pm 120 \mathrm{Mpa}$ y los todos los nodos en las dos direcciones están sujetos a un desplazamiento de $\pm 10 \mathrm{~mm}$. Las variables son seleccionadas de la siguiente lista $\mathrm{L}=\{113,2 ; 143,2 ; 145,9$; 174,$9 ; 185,9 ; 235,9 ; 265,9 ; 297,1 ; 308,6 ; 334,3$; $338,2 ; 497,8 ; 507,6 ; 736,7 ; 791,2 ; 1063,7\}\left(\mathrm{mm}^{2}\right)$. Las fuerzas en los nodos son P1 $=35 \mathrm{kN}, \mathrm{P} 2=35$ $\mathrm{kN}, \mathrm{P} 3=35 \mathrm{kN}$.

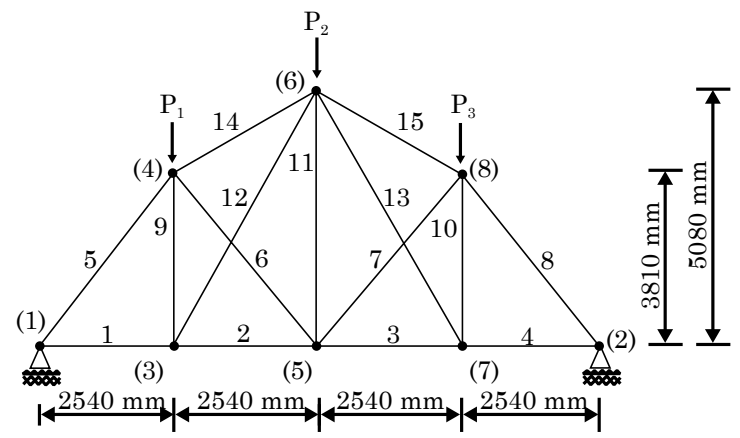

Fig. 3. Cercha plana de 15 barras Fuente: Dede [15]

La mejor solución encontrada por ASAM es $[113,2 ; 113,2 ; 113,2 ; 113,2 ; 736,7 ; 113,2 ; 113,2$; 736,$7 ; 113,2 ; 113,2 ; 113,2 ; 113,2 ; 113,2 ; 334,3$; $334,3] \mathrm{mm}^{2}$ con un peso mínimo de $105.735 \mathrm{~kg}$.

En la Fig. 4 se muestra la gráfica de convergencia y en el anexo Tabla 2 se comparan los resultados obtenidos con ASAM con técnicas como el PSO, $\mathrm{ACO}$, entre otros.

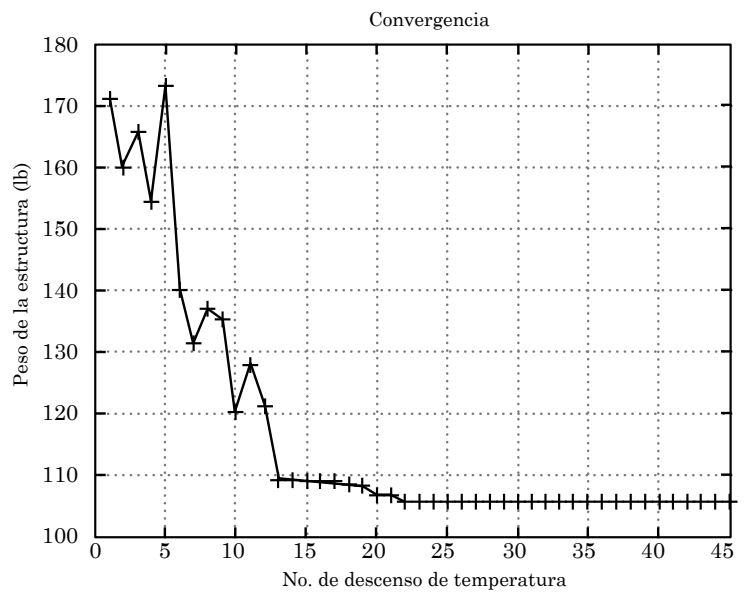

Fig. 4. Convergencia cercha plana de 15 barras Fuente: Autores 


\section{Cercha plana de 52 barras}

El tercer problema numérico considera el diseño óptimo de una cercha plana de 52 barras como se muestra en la Fig. 5. La densidad del material es de $7860,0 \mathrm{~kg} / \mathrm{m}^{3}$ y módulo de elasticidad de $207 \mathrm{GPa}$. Los 52 elementos son parametrizados en 12 grupos, de la siguiente manera: (1) A1-A4, (2) A5-A10, (3) A11-A13, (4) A14-A17, (5) A18-A23, (6) A24-A26, (7) A27-A30, (8) A31-A36, (9) A37-A39, (10) A40A43, (11) A44-A49, y (12) A50-A52. Todos los elementos tienen restricción a tensión y compresión de $180 \mathrm{MPa}$. La estructura es diseñada para dos cargas $\mathrm{PX}=100 \mathrm{kN}$ y PY $=200 \mathrm{kN}$. Las variables de diseño son seleccionadas del código AISC mostrado en el anexo Tabla 3.

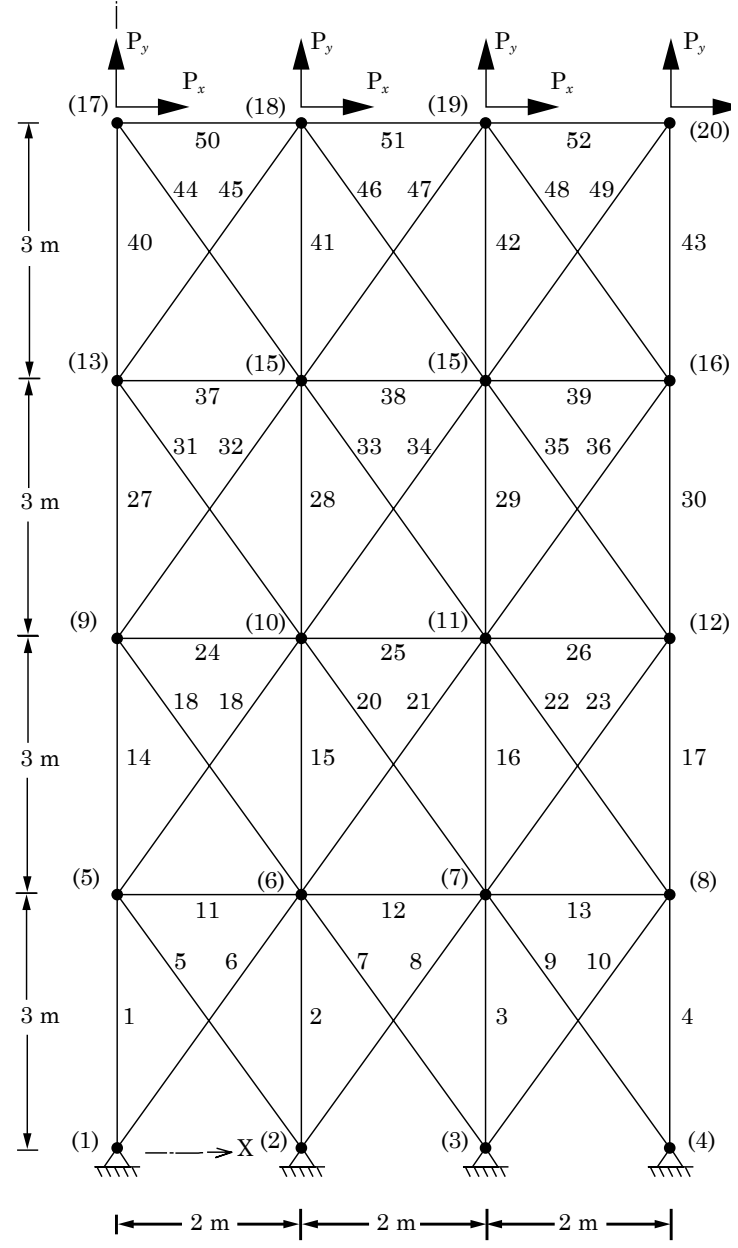

Fig. 5. Cercha plana de 52 barras Fuente: Kaveh [19]

En la Fig. 6 se presenta la gráfica de convergencia para este problema; además, en el anexo Tabla 4 se resumen los mejores diseños obtenidos por Sabohur [17], Kaveh [18,19] y Dede [15] junto con el mejor y peor diseño encontrado por ASAM.

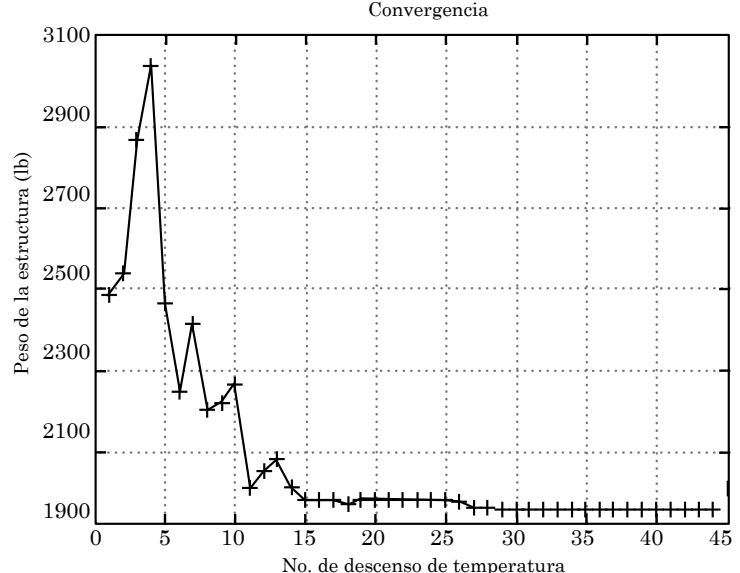

Fig. 6. Convergencia cercha plana de 52 barras Fuente: Autores

\section{CONCLUSIONES}

Se ha conseguido evaluar el desempeño del Algoritmo Simulated Annealing Modificado (ASAM) en el problema de minimización de peso de cerchas planas con variables discretas. Tres problemas clásicos tomados de la literatura fueron analizados para demostrar la eficiencia del algoritmo. Los valores de secciones transversales (áreas) y pesos fueron comparados con los reportados por otros autores, y mostraron que son coherentes y satisfactorios (ver anexos Tablas 1, 2 y 4), dando así validez al trabajo aquí presentado. En cuanto a la técnica empleada, se puede observar que ASAM tiene una versatilidad y robustez para enfrentar diversos tipos de problemas y con diferentes números de elementos. Esto se ve reflejado en los valores de peso y áreas de los elementos logrados.

\section{Financiación}

Artículo derivado del proyecto de investigación “Optimización estructural de cerchas en 2D”. Financiado por el grupo de investigación GRESA, Universidad de Sucre. Fecha de inicio: agosto de 2014. Fecha finalización: agosto de 2015.

\section{REFERENCIAS}

[1] Z.W. Geem, J.H. Kim, and G.V. Loganathan, "A new heuristic optimization algorithm: Harmony search", $\mathrm{Si}$ mulation, vol. 76, no. 2, pp. 60-68, 2001. http://dx.doi. org/10.1177/003754970107600201

[2] S. Kirkpatrick, C.D. Gelatt, and M.P. Vecchi, "Optimization by Simulated Annealing”, science, vol. 220, no. 4598, pp. 671-680, 1983.

[3] J. Holland, Adaptation in Natural and Artificial Systems. Massachusetts: The MIT press, 1975.

[4] D. E. Goldberg, Genetic algorithms in search optimization and machine learning. Boston, MA: Addison-Wesley, 1989. 


\section{ALGORITMO SIMULATED ANNEALING MODIFICADO (ASAM) PARA \\ MINIMIZAR PESO EN CERCHAS PLANAS CON VARIABLES DISCRETAS}

[5] M. Dorigo, V. Maniezzo, and A. Colorni, "The ant system: optimization by a colony of cooperating agents", Ieee Trans Syst Man Cybern, vol. B26, no. 1, pp. 29-41, 1996. http://dx.doi.org/10.1109/3477.484436

[6] J. Kennedy and R. Eberhart, "Particle swarm optimization," proc. Ieee int. Conf. Neural networks, vol. 4, pp. 1942-1948, 1995

[7] C. Millan, O. Begambre, and E. Millan, "Propuesta y validación de un algoritmo simulated annealing modificado para la solución de problemas de optimización", Rev. Int Métodos Numér. Cálc. Diseño ing., vol. 30, no. 4, pp. 264. 270,2014

[8] M. Sonmez, "Discrete optimum design of truss structures using artificial bee colony algorithm," struct. Multidisc optim., vol. 43, pp. 85-97, 2011

[9] K. A. Dowsland and B. A. Díaz, "Diseño de heuristica y fundamentos del Simulated Annealing", Revista Iberoamericana de Inteligencia Artificial, vol. 19, pp. 93-102, 2003.

[10] P. Capriles, L. Fonseca, H. Barbosa, and A. Lemonge, "Rank-based ant colony algorithms for truss weight minimization with discrete variables", Communications in Numerical Methods in Engineering, vol. 23, pp. 553-575, 2007.

[11] C. V. Camp, "Design of space trusses using big bang-big crunch optimization”, J. Struct. Eng. 2007, vol. 133, no. 7 pp. 999-1008, 2007.

[12] C. V. Camp and M. Farshchin, "Design of space trusses using modified teaching-learning based optimization", Engineering Structures, vol. 62-63, pp. 87-97, 2014. http://dx.doi.org/10.1016/j.engstruct.2014.01.020
[13] H. J. C. Barbosa, A. C. C. Lemonge, and C. C. H. Borges, "A genetic algorithm encoding for cardinality constraints and automatic variable linking in structural optimization”, Eng. Struct. 2008, vol. 30, no. 12, pp. 3708 3723,2008

[14] L. J Li, Z. B. Huang, and F. A. LIU, "A heuristic particle swarm optimization method for truss structures with discrete variables", Computer and Structures, vol. 87, pp. 435-443, 2009. http://dx.doi.org/10.1016/j. compstruc.2009.01.004

[15] T. Dede, "Application of teaching-learning-based-optimization algorithm for the discrete optimization of truss structures", Ksce journal of civil engineering, vol. 18, no. 6, pp. 1759-1767, 2014.

[16] Y. Zhang, J. Liu, B. Liu, C. Zhu, and Y. Li, "Application of improved hybrid genetic algorithm to optimize", j south china univ. Technol, vol. 33, no. 3, pp. 66-72, 2003.

[17] M.H. Sabour, H. Eskandar, and P. Salehi, "Imperialist competitive ant colony algorithm for truss structures", World applied Sciences Journal, vol. 12, no. 1, pp. 94105, 2011.

[18] A. Kaveh and S. Talatahari, "Particle swarm optimizer, ant colony strategy and harmony search scheme hybridized for optimization of truss structures", Computers and Structures, vol. 87, no. 5-6, pp. 267-283, 2009. http://dx.doi.org/10.1016/j.compstruc.2009.01.003

[19] A. Kaveh, B. Mirzaei, and A. Jafarvand, "An improved magnetic charged system search for optimization of truss structures with continuous and discrete variables", Applied soft computing, vol. 28, pp. 400-410, 2015. 
Anexos

Tabla 1. Comparación Diseño Óptimo para la Cercha Plana de 10 Barras.

\begin{tabular}{|c|c|c|c|c|c|c|c|c|c|c|}
\hline \multicolumn{9}{|c|}{ Áreas tranversales óptimas (in ${ }^{2}$} \\
\hline \multirow{2}{*}{ Variables } & Capriles [10] & Camp [11] & Barbosa [13] & Li [14] & Sonmez [8] & Dede [15] & Camp [12] & \multicolumn{3}{|c|}{ Este estudio } \\
\cline { 2 - 13 } & RBSA & BB-BC & AG & HPSO & ABC & TLBO & TBLO & ASAM $_{\text {peor }}$ & ASAM $_{\text {mejor }}$ \\
\hline 1 & $\mathbf{A}_{\mathbf{1}}$ & 33,50 & 33,50 & 33,50 & 30,00 & 33,50 & 33,50 & 33,50 & 33,50 & 33,50 \\
\hline 2 & $\mathbf{A}_{\mathbf{2}}$ & 1,62 & 1,62 & 1,62 & 1,62 & 1,62 & 1,62 & 1,62 & 1,62 & 1,62 \\
\hline 3 & $\mathbf{A}_{\mathbf{3}}$ & 22,90 & 22,90 & 22,90 & 22,90 & 22,90 & 22,90 & 22,90 & 22,90 & 22,90 \\
\hline 4 & $\mathbf{A}_{\mathbf{4}}$ & 14,20 & 14,20 & 14,20 & 13,50 & 14,20 & 14,20 & 14,20 & 14,20 & 14,20 \\
\hline 5 & $\mathbf{A}_{\mathbf{5}}$ & 1,62 & 1,62 & 1,62 & 1,62 & 1,62 & 1,62 & 1,62 & 1,62 & 1,62 \\
\hline 6 & $\mathbf{A}_{\mathbf{6}}$ & 1,62 & 1,62 & 1,62 & 1,62 & 1,62 & 1,62 & 1,62 & 1,62 & 1,62 \\
\hline 7 & $\mathbf{A}_{\mathbf{7}}$ & 7,97 & 22,90 & 22,90 & 7,97 & 7,97 & 7,97 & 22,90 & 7,97 & 7,97 \\
\hline 8 & $\mathbf{A}_{\mathbf{8}}$ & 22,90 & 7,97 & 7,97 & 26,50 & 22,90 & 22,90 & 7,97 & 22,90 & 22,90 \\
\hline 9 & $\mathbf{A}_{\mathbf{9}}$ & 22,00 & 1,62 & 1,62 & 22,00 & 22,00 & 22,00 & 1,62 & 22,00 & 22,00 \\
\hline 10 & $\mathbf{A}_{\mathbf{1 0}}$ & 1,62 & 22,00 & 22,00 & 1,80 & 1,62 & 1,62 & 22,00 & 1,62 & 1,62 \\
\hline Peso (lb) & $\mathbf{5 4 9 0 , 7 4 0}$ & $\mathbf{5 4 9 0 , 7 4 0}$ & $\mathbf{5 4 9 0 , 7 4 0}$ & $\mathbf{5 5 3 1 , 9 8 0}$ & $\mathbf{5 4 9 0 , 7 4 0}$ & $\mathbf{5 4 9 0 , 7 4 0}$ & $\mathbf{5 4 9 0 , 7 4 0}$ & $\mathbf{5 4 9 0 , 7 4 0}$ & $\mathbf{5 4 9 0 , 7 4 0}$ \\
\hline
\end{tabular}

Fuente: Autores

Tabla 2. Comparación Diseño Óptimo para la Cercha Plana de 15 Barras.

\begin{tabular}{|c|c|c|c|c|c|c|c|c|c|c|}
\hline \multicolumn{11}{|c|}{ Áreas transversales óptimas $\left(\mathrm{mm}^{2}\right)$} \\
\hline \multirow{2}{*}{\multicolumn{2}{|c|}{ Variables }} & \multirow{3}{*}{$\begin{array}{c}\text { Zhang [16] } \\
\text { HGA } \\
308,6\end{array}$} & \multicolumn{3}{|c|}{$\operatorname{Li}[14]$} & \multicolumn{2}{|c|}{ Eskandar [17] } & \multirow{3}{*}{$\begin{array}{c}\text { Dede [15] } \\
\text { TLBO } \\
113,2\end{array}$} & \multicolumn{2}{|c|}{ Este estudio } \\
\hline & & & \multirow{2}{*}{$\frac{\text { PSO }}{185,9}$} & \multirow{2}{*}{$\frac{\text { PSOPC }}{113,2}$} & \multirow{2}{*}{$\begin{array}{r}\text { HPSO } \\
113,2\end{array}$} & \multirow{2}{*}{$\frac{\text { ICACO }}{113,2}$} & \multirow{2}{*}{$\frac{\text { ICA }}{113,2}$} & & \multirow{2}{*}{$\frac{\mathrm{ASAM}_{\text {peor }}}{113,2}$} & \multirow{2}{*}{$\frac{\mathrm{ASAM}_{\text {mejor }}}{113,2}$} \\
\hline 1 & $\mathbf{A}_{1}$ & & & & & & & & & \\
\hline 2 & $\mathbf{A}_{2}$ & 174,9 & 113,2 & 113,2 & 113,2 & 113,2 & 113,2 & 113,2 & 113,2 & 113,2 \\
\hline 3 & $\mathbf{A}_{3}$ & 338,2 & 143,2 & 113,2 & 113,2 & 113,2 & 113,2 & 113,2 & 113,2 & 113,2 \\
\hline 4 & $\mathbf{A}_{4}$ & 143,2 & 113,2 & 113,2 & 113,2 & 113,2 & 113,2 & 113,2 & 113,2 & 113,2 \\
\hline 5 & $\mathbf{A}_{5}$ & 736,7 & 736,7 & 736,7 & 736,7 & 736,7 & 736,7 & 736,7 & 736,7 & 736,7 \\
\hline 6 & $\mathbf{A}_{6}$ & 185,9 & 143,2 & 113,2 & 113,2 & 113,2 & 113,2 & 113,2 & 113,2 & 113,2 \\
\hline 7 & $\mathbf{A}_{7}$ & 265,9 & 113,2 & 113,2 & 113,2 & 113,2 & 113,2 & 113,2 & 113,2 & 113,2 \\
\hline 8 & $\mathbf{A}_{8}$ & 507,6 & 736,7 & 736,7 & 736,7 & 736,7 & 736,7 & 736,7 & 736,7 & 736,7 \\
\hline 9 & $\mathbf{A}_{9}$ & 143,2 & 113,2 & 113,2 & 113,2 & 113,2 & 113,2 & 113,2 & 113,2 & 113,2 \\
\hline 10 & $\mathbf{A}_{10}$ & 507,6 & 113,2 & 113,2 & 113,2 & 113,2 & 113,2 & 113,2 & 113,2 & 113,2 \\
\hline 11 & $\mathbf{A}_{11}$ & 279,1 & 113,2 & 113,2 & 113,2 & 113,2 & 113,2 & 113,2 & 113,2 & 113,2 \\
\hline 12 & $\mathbf{A}_{12}$ & 174,9 & 113,2 & 113,2 & 113,2 & 113,2 & 113,2 & 113,2 & 113,2 & 113,2 \\
\hline 13 & $\mathbf{A}_{13}$ & 297,1 & 113,2 & 185,9 & 113,2 & 113,2 & 113,2 & 113,2 & 113,2 & 113,2 \\
\hline 14 & $\mathbf{A}_{14}$ & 235,9 & 334,3 & 334,3 & 334,3 & 334,3 & 334,3 & 334,3 & 334,3 & 334,3 \\
\hline 15 & $\mathbf{A}_{15}$ & 265,9 & 334,3 & 334,3 & 334,3 & 334,3 & 334,3 & 334,3 & 334,3 & 334,3 \\
\hline & (kg) & 142,117 & 108,840 & 108,960 & 105,735 & 105,735 & 105,735 & 105,735 & 105,735 & 105,735 \\
\hline
\end{tabular}

Fuente: Autores 
Tabla 3. Áreas Transversales disponibles del Código Aisc.

\begin{tabular}{|c|c|c|c|c|c|c|c|c|c|c|c|}
\hline No & $\mathrm{in}^{2}$ & $\mathbf{m m}^{2}$ & No & $\mathrm{in}^{2}$ & $\mathbf{m m}^{2}$ & No & in $^{2}$ & $\mathbf{m m}^{2}$ & No & $\mathrm{in}^{2}$ & $\mathbf{m m}^{2}$ \\
\hline 1 & 0,111 & 71,613 & 17 & 1,563 & 1008,4 & 33 & 3,84 & 2477,4 & 49 & 11,5 & 7419,3 \\
\hline 2 & 0,141 & 90,968 & 18 & 1,62 & 1045,2 & 34 & 3,87 & 2496,8 & 50 & 13,5 & 8709,7 \\
\hline 3 & 0,196 & 126,451 & 19 & 1,8 & 1161,3 & 35 & 3,88 & 2503,2 & 51 & 13,9 & 8967,7 \\
\hline 4 & 0,25 & 161,29 & 20 & 1,99 & 1283,9 & 36 & 4,18 & 2696,8 & 52 & 14,2 & 9161,3 \\
\hline 5 & 0,307 & 198,064 & 21 & 2,13 & 1374,2 & 37 & 4,22 & 2722,6 & 53 & 15,5 & 10000 \\
\hline 6 & 0,391 & 252,26 & 22 & 2,38 & 1535,5 & 38 & 4,49 & 2896,8 & 54 & 16 & 10323 \\
\hline 7 & 0,442 & 285,16 & 23 & 2,62 & 1690,3 & 39 & 4,59 & 2961,3 & 55 & 16,9 & 10903 \\
\hline 8 & 0,563 & 363,23 & 24 & 2,63 & 1696,8 & 40 & 4,8 & 3096,8 & 56 & 18,8 & 12129 \\
\hline 9 & 0,602 & 388,39 & 25 & 2,88 & 1858,1 & 41 & 4,97 & 3206,4 & 57 & 19,9 & 12839 \\
\hline 10 & 0,766 & 494,19 & 26 & 2,93 & 1890,3 & 42 & 5,12 & 3303,2 & 58 & 22 & 14194 \\
\hline 11 & 0,785 & 506,45 & 27 & 3,09 & 1993,5 & 43 & 5,74 & 3703,2 & 59 & 22,9 & 14774 \\
\hline 12 & 0,994 & 641,29 & 28 & 3,13 & 2019,4 & 44 & 7,22 & 4658,1 & 60 & 24,5 & 15806 \\
\hline 13 & 1 & 645,16 & 29 & 3,38 & 2180,6 & 45 & 7,97 & 5141,9 & 61 & 26,5 & 17097 \\
\hline 14 & 1,228 & 792,26 & 30 & 3,47 & 2238,705 & 46 & 8,53 & 5503,2 & 62 & 28 & 18064 \\
\hline 15 & 1,266 & 816,77 & 31 & 3,55 & 2290,3 & 47 & 9,3 & 6000 & 63 & 30 & 19355 \\
\hline 16 & 1,457 & 940 & 32 & 3,63 & 2341,9 & 48 & 10,85 & 7000 & 64 & 33,5 & 21613 \\
\hline
\end{tabular}

Fuente: Kaveh [19]

Tabla 4. Comparación Diseño Óptimo para la Cercha Plana de 52 Barras.

\begin{tabular}{|c|c|c|c|c|c|c|c|c|c|}
\hline \multicolumn{10}{|c|}{ Áreas transversales óptimas $\left(\mathrm{mm}^{2}\right)$} \\
\hline \multirow{2}{*}{\multicolumn{2}{|c|}{ Variables }} & \multicolumn{2}{|c|}{ Sabohur [17] } & \multirow{3}{*}{$\frac{\frac{\text { Kaveh [18] }}{\text { DHPSACO }}}{4658,055}$} & \multirow{3}{*}{$\begin{array}{c}\frac{\text { Dede [15] }}{\text { TLBO }} \\
4658,055\end{array}$} & \multicolumn{2}{|c|}{ Kaveh [19] } & \multicolumn{2}{|c|}{ Este estudio } \\
\hline & & \multirow{2}{*}{$\frac{\text { ICACO }}{4658,055}$} & \multirow{2}{*}{$\frac{\text { ICA }}{4658,055}$} & & & \multirow{2}{*}{$\frac{\text { MCSS }}{4658,055}$} & \multirow{2}{*}{$\frac{\text { IMCSS }}{4658,055}$} & \multirow{2}{*}{$\frac{\mathrm{ASAM}_{\text {peor }}}{4658,055}$} & \multirow{2}{*}{$\frac{\mathrm{ASAM}_{\text {mejor }}}{4658,055}$} \\
\hline 1 & $\mathbf{A}_{1}-\mathbf{A}_{4}$ & & & & & & & & \\
\hline 2 & $\mathbf{A}_{5}-\mathbf{A}_{10}$ & 1161,288 & 1161,288 & 1161,288 & 1161,288 & 1161,288 & 1161,288 & 1161,288 & 1161,288 \\
\hline 3 & $\mathbf{A}_{11}-\mathbf{A}_{13}$ & 494,193 & 363,225 & 494,193 & 494,193 & 363,225 & 494,193 & 494,193 & 494,193 \\
\hline 4 & $\mathbf{A}_{14}-\mathbf{A}_{17}$ & 3303,219 & 3303,219 & 3303,219 & 3303,219 & 3303,219 & 3303,219 & 3303,219 & 3303,219 \\
\hline 5 & $\mathbf{A}_{18}-\mathbf{A}_{23}$ & 939,998 & 939,998 & 1008,385 & 939,998 & 939,998 & 939,998 & 939,998 & 939,998 \\
\hline 6 & $\mathbf{A}_{24}-\mathbf{A}_{26}$ & 494,193 & 494,193 & 285,161 & 494,193 & 506,451 & 494,193 & 494,193 & 494,193 \\
\hline 7 & $\mathbf{A}_{27}-\mathbf{A}_{30}$ & 2238,705 & 2238,705 & 2290,318 & 2238,705 & 2238,705 & 2238,705 & 2238,705 & 2238,705 \\
\hline 8 & $\mathbf{A}_{31}-\mathbf{A}_{36}$ & 1008,385 & 1008,385 & 1008,385 & 1008,385 & 1008,385 & 1008,385 & 1008,385 & 1008,385 \\
\hline 9 & $\mathbf{A}_{37}-\mathbf{A}_{39}$ & 494,193 & 641,289 & 388,386 & 494,193 & 388,386 & 494,193 & 494,193 & 494,193 \\
\hline 10 & $\mathbf{A}_{40}-\mathbf{A}_{43}$ & 1283,868 & 1283,868 & 1283,868 & 1283,868 & 1283,868 & 1283,868 & 1283,868 & 1283,868 \\
\hline 11 & $\mathbf{A}_{44}-\mathbf{A}_{49}$ & 1161,288 & 1161,288 & 1161,288 & 1161,288 & 1161,288 & 1161,288 & 1161,288 & 1161,288 \\
\hline 12 & $\mathbf{A}_{50}-\mathbf{A}_{52}$ & 494,193 & 494,193 & 506,451 & 494,193 & 729,031 & 494,193 & 494,193 & 494,193 \\
\hline \multicolumn{2}{|c|}{ Peso (kg) } & 1902,605 & 1903,366 & 1904,830 & 1902,605 & 1904,050 & 1902,605 & 1902,605 & 1902,605 \\
\hline
\end{tabular}

Fuente: Autores 